\title{
Transtorno afetivo bipolar em paciente gestante com trágico desfecho fetal: relato de caso
}

Bipolar affective disorder in pregnant patient with tragic fetal outcome: case report Trastorno afectivo bipolar en paciente embarazada con resultado técnico fetal: reporte de caso Marina Brito PREVIDELLI 1 Amanda Oliva SPAZIANI 1

Talita Costa BARBOSA 1 Júlia Groto RICO'1

Iasmim Alves De Lima CUSTóDIO ${ }^{1}$ Ariany Aparecida Bonfim SIMIONI ${ }^{1}$ Raissa Silva FROTA 1

Rúbia Carla da Cunha SANTANA 3

Matheus Magalhães AZARIAS ${ }^{3}$ Osny Renato Martins LUZ ${ }^{4}$

Morisa Martins Leão CARVALHO ${ }^{4}$ Márcia Zucchi VIDOTTI ${ }^{5}$

Amanda De Azevedo Soares CARENO

${ }^{1}$ Curso de Medicina, Universidade Brasil, UB, 15600-000, Fernandópolis, São Paulo, Brasil

${ }^{2}$ Curso de Medicina, Universidade de Rio Verde, UniRV, 75901-970, Goianésia, Goiás, Brasil

${ }^{3}$ Médico residente de ginecologia e obstetrícia, Hospital Ensino Santa Casa de Misericórdia, SCF, 15600-000, Fernandópolis, São Paulo, Brasil ${ }^{4}$ Médico ginecologista e obstetra, Hospital Ensino Santa Casa de Misericórdia, SCF, 15600-000, Fernandópolis, São Paulo, Brasil ${ }_{5}^{5}$ Médica psiquiatra docente do curso de Medicina, Universidade Brasil, UB, 15600-000, Fernandópolis, São Paulo, Brasil

\section{Resumo}

O Transtorno Afetivo Bipolar (TAB) envolve a psicose, depressão e mania caracterizado por períodos de bom humor e irritação ou depressão, podendo trazer prejuízos para as relações afetivas. No caso de TAB em gestação o tratamento torna-se complicado, pois a escassez de estudos faz com que a terapêutica farmacológica seja suspensa. Sexo feminino, 37 anos. Em tratamento para transtorno afetivo bipolar há 7 anos. Paciente engravidou em 2018 em uso de: Risperidona; Citalopram; Biperideno; Carbonato de lítio; Clorpromazina; Propanolol; Brometazina; Clonazepam. Devido a gestação, descoberta com 20 semanas gestacionais, alterou-se a medicação. Fazia uso de: Clonazepam, Prometazina, Carbonato de Lítio, Haloperidol e Clorpromazina. Com 26 semanas gestacionais, ultrassom gestacional apresentava polidrâmnio. Com 30 semanas gestacionais apresentou dor abdominal de grande intensidade e foi internada. Na manhã seguinte apresentava melhora do quadro álgico, porém tinha 9 polpas digitais de dilatação, colo fino e anterior. Encaminhada para o centro cirúrgico para realização de cesárea com recém-nascido bradicardico e hipotônico que evoluiu com óbito seis dias após. A prescrição e uso de medicamentos na gestação, merece especial cuidado. Os trabalhos na área com gestantes são bastantes escassos, uma vez que estudos nesta fase da vida vão de encontro a princípios da bioética.

Descritores: Psiquiatria; Gestantes; Transtorno Bipolar.

\section{Abstract}

Bipolar Affective Disorder (BAD) involves psychosis, depression and mania characterized by periods of good mood and irritation or depression, which can cause damage to affective relationships. In the case of BAD in pregnancy, treatment becomes complicated, as the scarcity of studies causes pharmacological therapy to be discontinued. Female, 37 years old. In treatment for bipolar affective disorder for 7 years. Patient became pregnant in 2018 using: Risperidone; Citalopram; Biperiden; Lithium carbonate; Chlorpromazine; Propanolol; Bromethazine; Clonazepam. Due to pregnancy, discovered at 20 gestational weeks, the medication was changed. It used: Clonazepam, Promethazine, Lithium Carbonate, Haloperidol and Chlorpromazine. At 26 gestational weeks, gestational ultrasound had polyhydramnios. At 30 weeks of gestation she had severe abdominal pain and was hospitalized. In the morning, she presented pain improvement, but had 9 digital dilation pulps, thin and anterior neck. She was referred to the operating room for cesarean section with bradycardic and hypotonic newborn who died after six days. The prescription and use of medications in pregnancy deserves special care. Work in the area with pregnant women is quite scarce, since studies at this stage of life go against the principles of bioethics.

Descriptors: Psychiatry; Pregnant Women; Bipolar Disorder.

\section{Resumen}

El trastorno afectivo bipolar (BAD) implica psicosis, depresión y manía caracterizada por períodos de buen humor e irritación o depresión, que pueden dañar las relaciones afectivas. En el caso de BAD en el embarazo, el tratamiento se vuelve complicado, ya que la escasez de estudios hace que se suspenda la terapia farmacológica. Mujer de 37 años. En tratamiento por trastorno afectivo bipolar durante 7 años. La paciente quedó embarazada en 2018 usando: risperidona; Citalopram; Biperiden; Carbonato de litio; Clorpromazina; Propanolol; Brometazina; Clonazepam Debido al embarazo, descubierto a las 20 semanas de gestación, se cambió el medicamento. Utilizó: clonazepam, prometazina, carbonato de litio, haloperidol y clorpromazina. A las 26 semanas de gestación, la ecografía gestacional tenía polihidramnios. A las 30 semanas de gestación tuvo dolor abdominal intenso y fue hospitalizada. Por la mañana, presentó mejoría del dolor, pero tenía 9 pulpas digitales de dilatación, cuello delgado y anterior. Fue derivada al quirófano para una cesárea con un recién nacido bradicardia e hipotónico que murió después de seis días. La prescripción y el uso de medicamentos en el embarazo merece un cuidado especial. El trabajo en el área con mujeres embarazadas es bastante escaso, ya que los estudios en esta etapa de la vida van en contra de los principios de la bioética.

Descriptores: Psiquiatría; Mujeres Embarazadas; Trastorno Bipolar.

\section{INTRODUÇÃOO}

De acordo com o Manual Diagnóstico e Estatístico de Transtornos Mentais - DSM-V, o Transtorno Afetivo Bipolar (TAB) é um transtorno que envolve a psicose, depressão e mania. Tal transtorno anteriormente denominado como maníacodepressivo, por possuir particularidades do grupo das esquizofrenias e da depressão, é um transtorno mental caracterizado por períodos de bom humor e irritação ou depressão, podendo trazer prejuízos para as relações afetivas. $\mathrm{O}$ TAB é silencioso, causa mudanças repentinas de comportamento com apresentação de episódios de mania e hipomania ${ }^{1}$.

Existe diferença na manifestação da doença em gênero. Evidências apontam que mulheres com TAB tendem a evoluir com ciclagens rápidas, episódios depressivos repetitivos e em maior 
quantidade, mania disfórica em maior grau e hipomania mista, característica de estados mistos do tipo II ${ }^{2}$.

Nos episódios de euforia ou estado de mania, observa-se satisfação e felicidade patológica e artificial, percebe-se elevação do estado de ânimo, aceleração do curso do pensamento, loquacidade, vivacidade da mímica, gesticulações, risos, logorréia e confiança. Os episódios depressivos podem ser caracterizados por cinco ou mais sintomas como a apatia, desinteresse, perda do prazer, tendência ao isolamento, sensação de inutilidade, culpa excessiva, distúrbio do sono e distúrbio alimentar, entre outros, dificultando muito a convivência, além da ideia recorrente de morte ou suicídio ${ }^{3}$.

É bastante complicado definir os motivos das diferentes evoluções da doença nos dois gêneros. As teorias se baseiam desde contextos biopsicossocioculturais até características fisiológicas centradas no sistema hormonal e eixo tireoidiano ${ }^{4}$.

De acordo com a Sociedade Brasileira de Psiquiatria, existem três tipos de $\mathrm{TAB}$, sendo eles: tipo I, no qual os pacientes apresentam pelo menos um episódio maníaco e períodos de depressão profunda, tipo II, no qual os pacientes nunca apresentam episódios maníacos completos, mas ocorrem períodos de elevados níveis de energia e impulsividade que não são tão intensos como os da mania e esses episódios se alternam com episódios de depressão, já a ciclotimia é uma forma leve do transtorno que envolve oscilações de humor menos graves, pacientes com essa forma alternam entre hipomania e depressão leve ${ }^{5}$.

As pessoas que sofrem deste transtorno vivem com percepção alterada da realidade e podem demonstrar perda significativa de sensibilidade e sentimentos. Aproximadamente desses pacientes tentam suicídio, e os portadores de mania mista parecem apresentar risco maior. A taxa de suicídio é em torno de $15 \%$ para os não tratados ${ }^{6}$.

O TAB apresenta alta comorbidade com outros transtornos. A prevalência ao longo da vida de comorbidade de TAB e abuso de substâncias é de $42 \%$, com transtornos de ansiedade é de $42 \%$ e com transtornos alimentares é de $17 \%$. O paciente que tem TAB e abuso de substâncias apresenta características especiais, como maior taxa de estados mistos, ciclagem rápida, recuperação mais lenta, mais hospitalizações, mais tentativas de suicídio e início mais precoce do transtorno ${ }^{6}$.

No $\mathrm{TAB}$, entre os principais objetivos da terapêutica, está a diminuição dos sintomas agudos e a gravidade das alterações de comportamento do indivíduo, ocasionadas pelo transtorno. $\mathrm{O}$ tratamento consiste em estratégias farmacológicas e não farmacológicas. As primeiras englobam os medicamentos estabilizadores do humor, visando a fase aguda, prevenção de novos episódios e têm um papel importante na reparação da plástica sináptica, compensando uma série de alterações estruturais e funcionais em determinadas regiões do cérebro, provocadas pelas recaídas. Entre as terapêuticas farmacológicas mais utilizadas estão os estabilizadores do humor, como o lítio, antipsicóticos atípicos e antidepressivos ${ }^{7}$.

No tratamento específico do episódio maníaco, há preferência por lítio, valproato e carbamazepina. A combinação de antipsicóticos com o lítio ou o valproato pode ser mais efetiva. Como antipsicóticos atípicos, a olanzapina e a risperidona foram referenciados ${ }^{7}$.

Quanto às terapêuticas não farmacológicas indicadas no tratamento do $\mathrm{TAB}$, encontra-se a psicoeducação para manter a pessoa com transtorno inserida na sociedade. Ela envolve o provimento de informações a essas pessoas e familiares sobre o transtorno e o tratamento. O principal objetivo da intervenção psicoeducativa é capacitar o indivíduo com TAB a se apropriar de seu transtorno, possibilitando assim que lidem de forma promissora com as consequências. Outra terapêutica com resultados positivos no tratamento da TAB é a terapia cognitivo-comportamental (TCC). Ela é breve e estruturada, orientada para a solução de problemas e envolve a colaboração ativa entre a pessoa e o terapeuta para atingir objetivos estabelecidos ${ }^{7,8}$.

No caso de TAB em gestação o tratamento torna-se extremamente complicado, pois a escassez de estudos sobre os efeitos adversos para o binômio mãe/feto faz com que a terapêutica farmacológica muitas vezes seja suspensa pela falta de biossegurança, já que a maioria dos fármacos apresenta risco B, C e D de acordo com a classificação feita pela Food and Drug Administration (FDA), órgão norte-americano que controla alimentos e fármacos ${ }^{2}$.

CASO CLÍNICO

Sexo feminino, 37 anos, casada, com duas gestações, sendo dois partos cesáreos, sem abortos, gestante. Em tratamento psiquiátrico, com diagnóstico de transtorno afetivo bipolar há 7 anos. Paciente iniciou o tratamento em centro de atenção psicossocial no ano de 2013 após internação por tentativa de suicídio em hospital psiquiátrico, mantinha a prescrição de Citalopram $20 \mathrm{mg}$, Prometazina $25 \mathrm{mg}$, Carbamazepina $200 \mathrm{mg}$, Carbonato de Lítio $300 \mathrm{mg}$, Diazepam $10 \mathrm{mg}$, Nitrazepam 5mg, Clorpromazina 100mg, Ziprasidona $80 \mathrm{mg}$ e Biperideno $2 \mathrm{mg}$. Nessa situação apresentava alucinações auditivas e visuais além de inquietação. Manifestava ideação suicida, discurso negativo e pensamentos agressivos relacionados aos filhos. A relação familiar da paciente se mostrava conturbada. O marido, ex-usuário de drogas, contribuiu demasiadamente para o estresse da paciente; um dos filhos saiu recentemente da prisão, onde estava detido 
por tráfico de drogas. Relata possuir boa relação com a mãe e o outro filho e declara estar feliz com a gestação atual. Seguiu o tratamento de forma supervisionada passando por manejo em regime intensivo e mais duas internações até o ano 2018, quando engravidou novamente em uso de: Risperidona 80mg; Citalopram 20mg; Biperideno 2mg; Carbonato de lítio 300mg; Clorpromazina 100mg; Propanolol 40mg; Brometazina 25mg; Clonazepam 2mg. A medicação era assistida diariamente na Unidade de Saúde. A paciente apresentava-se lentificada, de humor deprimido, afeto embotado, autocuidado preservado e com alucinações auditivas e visuais. Relatava dependência severa de benzodiazepínicos e fobia excessiva.

Devido a gestação, descoberta com 20 semanas gestacionais, alterou-se a medicação. Fazia uso de: Clonazepam $2 \mathrm{mg}$, Prometazina $25 \mathrm{mg}$, Carbonato de Lítio 300mg, Haloperidol 5mg e Clorpromazina 100mg. Encontrava-se hipotímica, com afeto congruente; organizada; sem delírios e alucinações; com ideação suicida e autocuidado preservado. Foi orientada a diminuir a dose de Clonazepam, no entanto, apresenta extrema resistência. Com 26 semanas gestacionais, ultrassom gestacional apresentava-se com polidrâmnio. Com 30 semanas gestacionais apresentou dor abdominal de grande intensidade e foi encaminhada para o centro obstétrico, apresentava colo grosso, fechado e posterior ao toque e cardiotocografia sem alterações. Entretanto, devido ao quadro clínico foi internada. $\mathrm{Na}$ manhã seguinte apresentava melhora do quadro álgico, porém tinha 9 polpas digitais de dilatação, colo fino e anterior. Encaminhada para o centro cirúrgico em caráter de urgência para realização de cesárea que transcorreu sem intercorrências, porém recém-nascido apresentava-se bradicardico e hipotônico, foi intubado imediatamente em mesa de reanimação neonatal e evoluiu com óbito seis dias após.

\section{DISCUSSÃO}

A prescrição e uso de medicamentos na gestação, merece especial cuidado pelos profissionais que prestam assistência. Tal conduta merece atenção pelos riscos potenciais ao feto em desenvolvimento, devendo a princípio ser evitado. Dessa maneira, a utilização dos medicamentos deve ser vista com cautela e estar sujeita a avaliação criteriosa dos riscos e benefícios, devido as complicações sobre a saúde do feto ${ }^{9}$.

Os trabalhos na área com gestantes são bastantes escassos, uma vez que estudos nesta fase da vida vão de encontro a princípios da bioética e a maioria dos medicamentos atuais liberados $\mathrm{e}$ indicados para uso não são próprios e totalmente seguros contra efeitos colaterais e teratogênicos ${ }^{10,11}$.

Dentre as particularidades de cada classe de fármacos utilizados no tratamento de transtornos psiquiátricos temos as estratificações variando de $\mathrm{B}$ a $\mathrm{D}$, sendo que o risco $\mathrm{B}$ não possui evidência de risco em humanos ou os achados em animais demonstram risco, mas os achados em humanos não, ou se estudos adequados em humanos não têm sido realizados, achados em animais são negativos. Já o risco $\mathrm{C}$ diz que o risco não pode ser excluído; faltam estudos em humanos, e os estudos em animais são positivos para o risco fetal ou estão ausentes também. Contudo, potenciais benefícios podem justificar o risco potencial. Enquanto o risco D apresenta evidência positiva de risco, onde dados de investigação ou relatados, posteriormente, mostram risco ao feto, ainda assim, potenciais benefícios podem ter mais valor que o risco em potencial ${ }^{12,13}$.

Tenta-se preconizar a administração de medicamentos, durante a gravidez ou aleitamento materno, somente quando são necessários e em menor período possível. O primeiro trimestre é o mais complicado. Durante esse período a medicação é reduzida ou até mesmo descontinuada, consoante a gravidade da doença. $\mathrm{O}$ risco depende do medicamento prescrito, mesmo com os efeitos na gravidez sendo desconhecidos. Existem alguns fármacos, que são um pouco mais seguros para serem administrados. Um exemplo são os estabilizadores de humor, os quais devem ser seguidos regularmente em consulta obstétrica. Recomenda-se realizar ecografia ao feto regularmente, no intuito de eliminar qualquer suspeita de malformação ${ }^{14}$.

Nas gestantes, não há como privar dos riscos do uso de medicamentos, a não ser pela suspensão total do uso de fármacos, o que seria inadequado, visto que como a maioria da população, a gestante também está sujeita a intercorrências que podem gerar condutas terapêuticas medicamentosas. Existe sim a conduta de poupá-las da exposição aos riscos desnecessários. Para atenuar essa prática, é proposto o envolvimento responsável dos profissionais de saúde, para que medidas de intervenção sejam tomadas, promovendo uma utilização racional dos medicamentos ${ }^{15}$.

\section{REFERÊNCIAS}

1. Silva RA, Mograbi DC, Camelo EVM, Amadeo LN, Santana CMT, Landeira-Fernandez J et al . The relationship between insight and affective temperament in bipolar disorder: an exploratory study. Trends Psychiatry Psychother. 2018;30(3):210-15.

2. Dias RS, Kerr-Corrêa F, Torresan RC, Santos CHR. Transtorno bipolar do humor e gênero. Rev. psiquiatr. clín. 2006;33(2):80-91.

3. Moreno DH, Moreno RA. Tratamento de episódios maníacos e mistos. Debates em Psiquiatria. 2011;1(6):6-17.

4. Camacho RS, Cantinelli FS, Ribeiro CS, Cantilino 
A, Gonsales BK, Braguittoni E et al. Transtornos psiquiátricos na gestação e no puerpério: classificação, diagnóstico e tratamento. Rev Psiq Clín. 2006;33(2);92-102.

5. Campos LKS, Seidinger-Leibovitz FM, Santos Junior A, Turato ER. O transtorno bipolar como experiência: a perspectiva dos filhos. $\mathbf{J}$ bras psiquiatr. 2018;67(1):39-44.

6. Costa AMN. Transtorno afetivo bipolar: carga da doença e custos relacionados. Rev Psiq Clín. 2008;35(3):104-10.

7. Santos VC, Silva RC, Mochizuki AB, Anjos KF. Transtorno afetivo bipolar: terapêuticas, adesão ao tratamento e assistência de enfermagem. REBRASF. 2017;1(1):10-21.

8. Silva, LOL, Dias CA, Rosalino FU. Processos terapêuticos no tratamento do transtorno afetivo bipolar: revisão integrativa. Rev Psicol Saúde. 2017;9(3):63-6.

9. Melo SCCS, Pelloso SM, Carvalho MDB, Oliveira NLB. Uso de medicamentos por gestantes usuárias do Sistema Único de Saúde. Acta paul enferm. 2009;22(1):66-70.

10. Cordeiro, LP. Consumo de substâncias psicoativas por gestantes [monografia]. Campina Grande: Centro de Ciências Biológicas e da Saúde CCBS UESPB; 2018.

11. ADEB, Associação de Apoio Aos Doentes Depressivos e Bipolares-. Doença bipolar e gravidez. O que preciso saber?: Informação para doentes e familiares. 2019.

12. Murta NNR, Guimarães CFB, Martins TMD, Cunha RG. Perfil gestacional e exposição fetal a drogas de abuso. Rev NBC. 2018;8(16):74-87.

13. Salim M, Sharma V, Anderson KK. Recurrence of bipolar disorder during pregnancy: a systematic review. Arch Womens Ment Health. 2018;21(4):475-79.

14. Hatters Friedman S, Moller-Olsen C, Prakash C, North A. Atypical antipsychotic use and outcomes in an urban maternal mental health service. Int $\mathbf{J}$ Psychiatry Med. 2016;51(6):521-33.

15. Byatt N, Cox L, Moore Simas TA, Biebel K, Sankaran P, Swartz HA, Weinreb L. Access to pharmacotherapy amongst women with bipolar disorder during pregnancy: a preliminary study. Psychiatr Q. 2018;89(1):183-90.

\section{CONFLITO DE INTERESSES}

Os autores declaram não haver conflitos de interesse.

\section{AUTOR PARA CORRESPONDÊNCIA}

\section{Amanda Oliva Spaziani}

E-mail: spazianimedicina@gmail.com
Submetido em 03/11/2019

Aceito em 07/10/2020 